\title{
0 papel do aleitamento materno, da dieta e do estado nutricional no desenvolvimento de asma e atopia*
}

\author{
The role of breastfeeding, diet and nutritional status \\ in the development of asthma and atopy
}

\author{
Aline Petter Schneider ${ }^{1}$, Renato Tetelbom Stein ${ }^{2}$, Carlos Cezar Fritscher ${ }^{3}$
}

\section{Resumo}

0 aumento na prevalência da asma e atopia, observado em diversas populações nos anos recentes, contribui para torná-las importantes problemas de saúde pública. A possível influência de fatores relacionados à nutrição humana tem sido demonstrada em crescente número de estudos. 0 entendimento do papel do aleitamento materno, da dieta, e do estado nutricional, particularmente da obesidade, assim como as respostas imunológicas desencadeadas, ajuda a melhorar a compreensão sobre a relação entre estresse oxidativo, inflamação brônquica, e o desenvolvimento de sintomas asmáticos e atópicos. Este artigo apresenta uma revisão da literatura publicada sobre os aspectos da relação entre nutrição, asma, e atopia, nas duas últimas décadas.

Descritores: Asma; Estado nutricional; Aleitamento materno; Dieta.

\begin{abstract}
In many populations, the prevalence of asthma and atopy has increased in recent years. As a result, both conditions have become major public health problems. The possible influence of nutrition-related factors has been demonstrated in an increasing number of studies. Information regarding the role of breastfeeding, diet, nutritional status (obesity in particular), as well as regarding the immunologic responses triggered, helps to improve our understanding of the correlation between oxidative stress, bronchial inflammation, and the development of atopic and asthma symptoms. The article presents a review of the published literature on the relationships established between and among nutrition, asthma, and atopy over the last two decades.
\end{abstract}

Keywords: Asthma; Nutritional status; Breast feeding; Diet.

\footnotetext{
* Trabalho realizado na Pontifícia Universidade Católica do Rio Grande do Sul - PUCRS - Porto Alegre (RS) Brasil.

1. Professora do Curso de Nutrição, Centro Universitário Metodista do Instituto Porto Alegre - IPA - Porto Alegre (RS) Brasil.

2. Professor Adjunto, Departamento de Pediatria da FAMED, Pontifícia Universidade Católica do RGS - PUCRS - Porto Alegre RS) Brasil.

3. Professor Titular, Departamento de Medicina Interna da FAMED, Pontifícia Universidade Católica do RGS - PUCRS - Porto Alegre (RS) Brasil.

Endereço para correspondência: Renato Tetelbom Stein. Pontifícia Universidade Católica do Rio Grande do Sul, Faculdade de Medicina, Departamento de Pediatria, Av. Ipiranga, 6690, conj. 420, Partenon, CEP 90610-001, Porto Alegre, RS, Brasil.

Tel 5551 3336-4211. Fax 5551 3336-2033. E-mail: rstein@pucrs.br

Recebido para publicação em 26/10/06. Aprovado, após revisão, em 3/11/06.
} 


\section{Introdução}

Nas últimas décadas, observou-se um importante aumento na prevalência da asma e da atopia, em diversas populações. ${ }^{(1)}$ Os aspectos genéticos e ambientais dessa ocorrência têm sido amplamente estudados. A asma é uma doença inflamatória crônica, caracterizada por hiperresponsividade das vias aéreas inferiores e por limitação variável ao fluxo aéreo, reversível espontaneamente ou com tratamento, enquanto a atopia é a resposta positiva a alérgenos ambientais comuns, medida através de testes alérgicos cutâneos, ou por níveis séricos de imunoglobulina E (lgE) específico a alérgenos. Recentemente, fatores dietéticos foram associados ao aumento da prevalência de asma e atopia. Dentre estes, destacam-se o impacto do aleitamento materno, a dieta, e o estado nutricional. No entanto, para muitas das associações entre diferentes aspectos de dieta, com asma e atopia, a compreensão é ainda limitada pela carência de evidências de uma relação causa-efeito. Isto ocorre pela dificuldade em se determinar o efeito longitudinal da dieta, especialmente quanto a seu impacto na incidência e na severidade da doença. Para delinear medidas preventivas, visando reverter o aumento da asma e da atopia, é desejável identificar quais fatores estão envolvidos no seu aparecimento. Neste artigo, será apresentada uma revisão dos temas publicados na literatura especializada, referentes à interação entre nutrição, asma e atopia. Foram realizadas buscas nos bancos de dados Medline e Literatura LatinoAmericana e do Caribe em Ciências da Saúde, usando os termos pré-especificados asma, atopia, dieta, nutrição, e estado nutricional.

\section{Interações mãe-filho}

0 ambiente intra-uterino é o primeiro ao qual o indivíduo é exposto. Exposições in utero têm potencial para causar efeitos duradouros, e funções orgânicas, como a respiratória, podem ser profundamente afetadas. ${ }^{(2)}$ Neste contexto, torna-se importante avaliar a maneira pela qual os hábitos da mãe, durante a gestação, podem interferir nos eventos de saúde futuros de seus filhos. Em relação à dieta, observou-se que o consumo materno de peixe durante a gravidez pode afetar o risco de asma na criança, ao modular o seu desenvolvimento imunológico. 0 tipo de peixe ingerido pode ser importante, pelas diferenças no conteúdo de ácidos graxos. Em um estudo de caso-controle realizado no sul da Califórnia (EUA), observou-se que a ingestão materna de óleo de peixe durante a gravidez pode proteger seus filhos contra a asma; contudo, consumir peixe processado empanado, uma fonte de gordura trans durante a gravidez, pode aumentar o risco de asma nas crianças. ${ }^{(3)}$

As observações sobre como o tipo de dieta da mãe pode interferir no desenvolvimento de doenças com fundo alérgico sugere o desenvolvimento de abordagens voltadas para prevenir doença atópica na criança. Uma possibilidade seria prescrever a dieta, evitando antígenos durante a gravidez, direcionada a mulheres com alto risco de dar à luz a uma criança atópica, com base na história de doença atópica na mãe, pai, ou em outro filho prévio. A evidência atual não sugere forte efeito protetor deste tipo de dieta na incidência de eczema atópico ou asma, pelo menos durante os primeiros 12-18 meses de vida. Os dados sobre rinite/conjuntivite alérgica e urticária são limitados a um único estudo cada, os quais são insuficientes para que se faça inferências significativas. Além disso, essas dietas especiais poderiam até causar efeitos indesejáveis na nutrição materna ou fetal. ${ }^{(4)}$

\section{Aleitamento materno}

0 aleitamento materno éo método de alimentação infantil preferido, por inúmeras razões. Entretanto, o papel desempenhado pelo aleitamento materno na ocorrência de doenças alérgicas, particularmente na asma, é controverso. Em alguns estudos, o aleitamento exclusivo, nos primeiros 6 meses de vida do lactente, mostrou redução no surgimento de alergias e asma, ${ }^{(5,6)}$ e diminuiu discretamente o risco de atopia. ${ }^{(7,8)}$ Em outros, foi encontrada associação positiva entre crianças amamentadas e desenvolvimento de alergias respiratórias, ${ }^{(9,10)}$ mas a ausência de qualquer efeito também foi observada. ${ }^{(11)}$

Há, também, dados conflitantes sobre o impacto do aleitamento materno no desenvolvimento de asma na infância tardia. Recentemente, um estudo australiano investigou a relação entre aleitamento e a prevalência de asma em crianças aos 14 anos: em uma coorte de 4.964 crianças, cujos dados estavam disponíveis, o aleitamento materno não aumentou nem diminuiu a prevalência de asma. ${ }^{(12)}$

Razões para esta controvérsia incluem diferenças e falhas metodológicas nos estudos realizados, a 
complexidade imunológica do leite materno e, possivelmente, diferenças genéticas entre os pacientes, que influenciam se o aleitamento materno protege contra o desenvolvimento de alergias ou, inversamente, sensibiliza. Em geral, fórmulas infantis isentas de leite de vaca ou de proteína de soja, comparadas ao leite materno, ocasionam maior incidência de dermatite atópica e doenças, na infância, com sibilância. ${ }^{(13)}$

Uma das principais razões pelas quais o efeito do aleitamento materno no desenvolvimento de alergias permanece ainda em discussão deve-se à complexidade da interação entre leite materno, milieu intestinal e sistema imune. Alguns elementos do leite materno têm papel protetor contra o desenvolvimento de alergias, enquanto outros atuam sensibilizando. Mesmo um baixo nível de exposição da mucosa, como a alérgenos inalatórios, pode induzir à secreção de anticorpos no leite materno, tanto de mães alérgicas como não alérgicas. ${ }^{(14)}$ Sabe-se que a secreção de $\lg A$ passa da mãe para o lactente via leite materno, ou colostro, ${ }^{(15)}$ e baixos níveis de $\lg$ A para ovoalbumina foram observados no colostro e leite maduro de mães alérgicas, quando comparadas a mães sem alergia. ${ }^{(14)}$ As concentrações de citocinas podem ter um papel na imunogenicidade do leite materno. As citocinas interleucina $1 \mathrm{~L}-4$, 1L-5, e lL-13, mais envolvidas com a produção de $\lg E$ e indução de resposta eosinofílica, estão presentes em alta concentração no leite materno de mães atópicas, comparadas com as concentrações de mães não atópicas. ${ }^{(16)} 0$ fator de crescimento transformador beta, uma das citocinas predominantes no leite humano, aumenta a capacidade do lactente de produzir lgA contra beta-lactoglobulina, caseina, gliadina, e ovoalbumina. ${ }^{(17)} 0$ CD14 solúvel, que está presente em altas concentrações no leite materno e tem importante papel na indução da resposta de linfócitos $\mathrm{T}$ auxiliares a bactérias, pode também proteger contra o desenvolvimento de alergias. ${ }^{(15)} 0$ alto nível de proteína eosinofílica catiônica no leite materno está associado à maior incidência de alergia à proteína do leite de vaca e à determatite atópica. ${ }^{(18)}$

Antígenos alimentares têm sido detectados no leite materno. Beta-lactoglobulina, caseína, e globulina bovina, foram detectados no leite materno de mulheres que não restringiam a sua ingestão de leite de vaca, ovos e trigo, durante o período de lactação. ${ }^{(19,20)}$ Alérgenos foram detec- tados no leite materno, entre 2 e $6 \mathrm{~h}$ depois da ingestão materna, mesmo 4 dias após a ingestão. Proteínas do amendoim também foram detectadas no leite materno. Crianças atópicas, já sensibilizadas por estes alimentos, podem ter exacerbação depois da ingestão do leite materno contendo estes alérgenos, e apresentam melhora da doença depois da restrição dos mesmos pela mãe. ${ }^{(21)}$ Mesmo que estes antígenos possam ser detectados, ainda é incerto se eles podem levar à sensibilização ou tolerância. Parece evidente, ao menos, que o aleitamento materno, por ao menos 4 meses, protege contra o desenvolvimento de dermatite atópica, em crianças com sibilância precoce. ${ }^{(7)}$

A ausência de aleitamento materno tem sido associada a várias desordens crônicas da infância, asma, doença celíaca, e obesidade. ${ }^{(22)}$ Como alguns estudos observaram associação entre aleitamento materno e maior risco de desenvolver asma e eczema, foi proposto que esse resultado possa ser devido ao fato de que sinais precoces de doença atópica na criança induzam a que as mães prolonguem o aleitamento. Isso poderia mascarar o efeito protetor do aleitamento, ou mesmo resultar na conclusão aparente de que o aleitamento constitui um fator de risco para o desenvolvimento de doenças atópicas, o que deve ser considerado nas futuras investigações sobre aleitamento materno e doenças atópicas. ${ }^{(23)}$

\section{Ingestão dietética}

Os pulmões são continuamente expostos a concentrações relativamente altas de oxigênio, representando, em comparação com outros órgãos, um tecido sensível aos efeitos oxidantes. Em certos momentos, durante a vida, esse desafio diário cresce exponencialmente. A primeira agressão oxidativa ocorre ao nascimento, quando as células são expostas ao repentino aumento de cinco vezes na concentração de oxigênio. A partir desse momento, o pulmão humano, da infância à velhice, está sujeito a eventos oxidativos danosos, como conseqüência de poluentes inalatórios, irritantes ambientais, e a várias doenças, incluindo asma, doença pulmonar obstrutiva crônica, e fibrose cística, entre outras..$^{(24)}$

A hipótese nutricional atribui o aumento das alergias respiratórias a mudanças nos hábitos de ingestão dietética, principalmente de antioxidantes e/ou lipídios. ${ }^{(25,26)}$ Antioxidantes e lipídios na dieta induzem mecanismos imunomodulatórios e pró- 
inflamatórios variados e complexos, com provável associação benéfica entre estes compostos e parâmetros de asma e doença atópica. ${ }^{(25)}$

Paralelamente, estudos com crianças e adultos mostram uma associação inversa entre ingestão de Vitaminas E e C com asma e atopia, conferindo a essas substâncias um possível papel protetor. ${ }^{(27,28)}$ No entanto, permanecem dúvidas sobre a potencial ação de determinados nutrientes, como os chamados imunomoduladores, principalmente quanto à possibilidade de prevenir ou minimizar o risco de asma e alergias respiratórias, através de intervenções com suplementação na dieta. Como a asma sintomática em adultos é associada à baixa ingestão de frutas, nutrientes antioxidantes, vitamina C e manganês, sugere-se que a dieta possa ser um fator de risco potencialmente modificável para o desenvolvimento de asma. ${ }^{(29)}$

Os padrões de dieta ocidentais tendem a uma deficiência em antioxidantes, fato esse que está, ao menos temporalmente, associado a um conseqüente aumento na prevalência das doenças respiratórias. A mudança na ingestão dietética de antioxidantes pode reduzir as defesas pulmonares, com aumento na susceptibilidade respiratória a danos oxidativos, resultando em inflamação respiratória e asma. ${ }^{(25)} \mathrm{Um}$ estudo transversal sobre a associação de nutrientes antioxidantes e marcadores de estresse oxidativo com volume expiratório forçado no primeiro segundo $\left(V_{E F}\right)$ e capacidade vital forçada (CVF), em pessoas com limitação crônica do fluxo aéreo, mostrou que vários nutrientes, como beta-criptoxantina, luteína, retinol, beta-caroteno, beta-criptoxantina, vitamina $\mathrm{C}$, e licopeno, foram positivamente associados ao $\mathrm{VEF}_{1}, \%$ do previsto, e CVF, enquanto a glutationa eritrocítica e substâncias ácidas reativas foram negativamente associadas. Os resultados apóiam a hipótese de que, ao menos em adultos, um desequilíbrio no status antioxidante/oxidante parece estar associado a algum grau de limitação crônica do fluxo aéreo, ao qual contribuem hábitos dietéticos e o estresse oxidativo. ${ }^{(30)}$

0 mecanismo pelo qual se desenvolve a sensibilização e a inflamação nas vias aéreas pode ser promovido pelo aumento da ingestão de ácidos graxos ômega-6, derivados de margarinas e óleos vegetais, pela diminuição da ingestão de ômega-3, de derivados de óleos de peixe, e diminuição na ingestão de antioxidantes (frutas e vegetais), o que contribuiria para o aumento da asma e atopia. ${ }^{(25)} \mathrm{Os}$ mais comuns ácidos graxos polinsaturados (AGPIs) são o ácido linoléico (ômega-6) e o ácido linolênico, os quais podem ser convertidos em AGPls de cadeia longa, por uma simples dessaturação. 0 ácido linoléico é convertido em ácido araquidônico, e pode ser metabolizado pela ciclooxigenase e enzima lipogenase em protaglandinas, tromboxanos, leucotrienos, e lipoxinas, todos associados à resposta inflamatória observada na asma e atopia. ${ }^{(31)}$ As associações com ácidos graxos n-6 e n-3 são complexas e podem diferir entre asma e dermatite atópica. A idéia é que a dermatite atópica esteja associada a um defeito enzimático no metabolismo lipídico. Entretanto, os resultados de intervenções com suplementação na dermatite já estabelecida não foram animadores. Há crescente interesse na hipótese de que a ingestão de antioxidantes e lipídios possa ser importante para modular a expressão da doença durante a gravidez e primeira infância, abrindo a possibilidade para intervenções dietéticas direcionadas a esses grupos. Também é muito provável que haja variação individual nas respostas à suplementação com lipídios e antioxidantes. ${ }^{(25)}$

Estudos transversais demonstram risco reduzido de asma associado à alta ingestão de frutas e vegetais. ${ }^{(32-34)} \mathrm{Um}$ estudo que investigou se a ingestão dietética prediz a prevalência de asma entre mulheres francesas, após ajustamento para idade, índice de massa corporal, status menopausal, fumo, ingestão calórica total, atividade física, e uso de suplementos dietéticos, mostrou que a maior ingestão de tomates, cenouras, e vegetais folhosos, induz menor prevalência de asma. Com a exceção de maçãs, nenhuma outra fruta foi associada à prevalência da asma. Os resultados sugerem que a ingestão de alguns vegetais pode diminuir a prevalência de asma no adulto. ${ }^{(34)}$ Admite-se que o alto consumo de maçãs possa proteger contra asma e doença pulmonar obstrutiva crônica, efeito atribuído ao conteúdo flavonóide. Em um estudo de caso-controle britânico, a ingestão de dietéticos de catequinas, flavonóis e flavonas (as três principais sub-classes de flavonóides) não foi associada à asma, severidade da asma ou produção crônica de catarro, após o controle de potenciais confundidores. É possível que outros flavonóides ou polifenóis, presentes em maçãs, possam explicar o efeito protetor das maçãs na doença pulmonar obstrutiva. ${ }^{(35)} \mathrm{Em}$ um estudo escandinavo, a ingestão de frutas frescas, ou vegetais, na infância, mas não de quantidades 
excedentes de vitaminas, ou suplementos de óleo de fígado de bacalhau, diminuiu o risco de asma. 0 uso precoce de suplementos de óleo de fígado de bacalhau, e extra de vitaminas, foi associado à maior sensibilização alérgica. A área de moradia também influenciou a sensibilização alérgica, com diferenças entre áreas costeiras e interiores. ${ }^{(36)}$

A vitamina C, extensivamente investigada, está associada em diversos estudos transversais e de casocontrole ao risco reduzido de asma, ${ }^{(32,33)}$ embora um estudo longitudinal sobre a ingestão de vitamina $C$ não tenha encontrado efeito na incidência de asma. ${ }^{(37)}$ Em ensaios randomizados, a vitamina C, combinada a outros antioxidantes, mostrou proteção contra broncoconstrição induzida por ozônio, ${ }^{(32)}$ mas a evidência do efeito da vitamina $C$, administrada isoladamente, é pouco conclusiva. Foi observado, entretanto, que as concentrações plasmática e leucocitária de vitamina $\mathrm{C}$ são siginificativamente menores em asmáticos do que em indivíduos saudáveis, e que há associação entre a duração da asma e níveis plasmáticos de vitamina C. ${ }^{(38)}$

Os efeitos da vitamina E têm sido menos estudados. Contudo, há alguma evidência relacionando vitamina $\mathrm{E}$ à asma, ${ }^{(32,33)}$ indicando uma associação inversa entre a ingestão dessa vitamina e a sensibilização alérgica e níveis séricos de $\lg \mathrm{E}$, em adultos, ${ }^{(28)}$ e de redução da incidência de asma. ${ }^{(37)}$ Como para a vitamina C, a vitamina E é efetiva quando administrada com outros antioxidantes, protegendo contra os efeitos do ozônio na asma, ${ }^{(32,33)}$ mas um recente ensaio clínico randomizado comparando a suplementação de vitamina E com placebo, por 6 semanas, em 72 pacientes com asma, não demonstrou benefício clínico. ${ }^{(27)}$
Carotenóides são potentes antioxidantes dietéticos, que podem proteger contra asma, reduzindo o dano oxidativo. A baixa concentração de vitamina A é detectada em várias doenças pediátricas. Contudo, as evidências para os efeitos de vitamina A e beta-caroteno também são limitadas, com alguns estudos transversais sugerindo efeito protetor, ${ }^{(32,33)}$ e um estudo longitudinal mostrando ausência de associação com a incidência de asma.. ${ }^{(37)}$ Sabe-se que os níveis plasmáticos de carotenóides refletem os níveis de carotenóides nas vias aéreas, e que o uso de suplementos orais melhora os níveis plasmáticos, o que é refletido nas vias aéreas. Observou-se, também, que os níveis de vitamina $A$, em crianças com asma, são significativamente menores do que em controles, e que a severidade da asma tem correlação negativa com os níveis séricos de vitamina A: crianças com asma grave persistente têm, marcadamente, menor nível sérico, em comparação aos com asma leve intermitente. ${ }^{(39)}$

Mudanças no estilo de vida, nas últimas décadas, são uma provável explicação para o aumento das doenças alérgicas nesse período. 0 consumo de fast food é relacionado à prevalência de asma e alergia, conforme um estudo transversal envolvendo 1.321 crianças, na Nova Zelândia. Após o ajustamento para fatores de estilo de vida, incluindo outras dietas e variáveis de índice de massa corporal, o consumo de hambúrguer (dose-dependente) foi um fator de risco independente para uma história de sibilância, comparado ao de crianças que nunca comeram hambúrgueres. Não foi observado efeito na atopia. ${ }^{(40)} \mathrm{A}$ Tabela 1 sumariza as possíveis influências no desenvolvimento da asma e alergia.

Tabela 1 - Nutrientes ou grupo de nutrientes implicados na etiologia da asma e seu mecanismo de efeito.

\begin{tabular}{ll}
\hline \multicolumn{1}{c}{ Nutriente(s) } & \multicolumn{1}{c}{ Papel e potencial mecanismo de efeito } \\
\hline Vitaminas A, C, e E & Antioxidante; proteção contra oxidação inflamatória endógena e exógena \\
Vitamina C & Inibição da prostaglandina \\
Vitamina E & Estabilização da membrana, inibição da produção de lgE \\
Flavonas e Flavonóides & Antioxidante, estabilização de mastócitos \\
Magnésio & Relaxamento do músculo liso, estabilização de mastócitos \\
Selênio & Antioxidante, cofator da glutationa peroxidase \\
Cobre, Zinco & Antioxidante, cofator da superoxide dismutase \\
n-3 (ácidos graxos) & Substituição do leucotrieno, estabilização das membranas inflamatórias \\
& celulares \\
n-6 polinsaturados/ácidos graxos trans & Aumento da produção de eicosanóides \\
Sódio & Aumento da contração muscular lisa \\
\hline
\end{tabular}

Revisado e reimpresso com permissão.(42) 


\section{Restrição calórica}

Estudos epidemiológicos sugerem uma correlação entre o aparecimento de asma e fatores dietéticos não alérgicos, especialmente dieta calórica elevada. Em um estudo com 38 pacientes, houve significante aumento no $\mathrm{VEF}_{1}$ e CVF, no grupo de restrição calórica, em comparação ao controle. Intervenções dietéticas, com programas de perda de peso, podem trazer benefícios a pacientes específicos. Contudo, o impacto de uma dieta de calorias controladas nos sinais e sintomas da asma, na população geral, precisa ser estabelecido. ${ }^{(41)}$

\section{Estado nutricional}

Observa-se, nos últimos anos, uma expansão de erros alimentares, de sedentarismo e de aumento de peso, em paralelo ao aumento das doenças associadas. ${ }^{(43,44)} \mathrm{A}$ obesidade se tornou um problema de saúde pública, nos Estados Unidos e em diversos países, o que inclui a prevalência de sobrepeso em crianças e adolescentes. ${ }^{(43)}$ No Brasil, o aumento da obesidade também é progressivo. ${ }^{(44)}$ Diversos estudos transversais têm demonstrado associação entre obesidade, asma, sintomas respiratórios, e hiperresponsividade brônquica. ${ }^{(45,46)}$ Entretanto, a associação positiva observada entre índice de massa corporal (IMC) elevado e asma não foi significativa para atopia e contagem total de eosinófilos séricos. ${ }^{(47)}$ Os dados sobre a influência da obesidade na severidade da asma são conflitantes. ${ }^{(45,46)}$ A relação é muito mais forte no sexo feminino. Em uma coorte de 1.000 indivíduos, o IMC aumentado estava associado à asma e atopia em mulheres, mas não em homens. ${ }^{(48)}$ Os achados em 9.552 participantes do European Community Respiratory Health Survey e do Swiss Cohort Study on Air Pollution and Lung Disease in Adults corroboram os relatos da maior associação entre asma e obesidade em mulheres do que em homens. ${ }^{(49)}$

A observação de que a perda de peso melhora a asma, e de que ratos obesos têm hiperresponsividade inata da via aérea e maior resposta a desencadeantes da asma, apóia a relação entre obesidade e asma. A base para essa relação é desconhecida, mas pode ser o resultado de etiologias em comum, comorbididades, efeitos da obesidade sobre o volume pulmonar, ou adipocinas. ${ }^{(45)}$ Estudos para elucidar a base genética de asma e obesidade identificaram polimorfismos em regiões específicas dos cromos- somos $5 q, 6 p, 11 q 13$, e 12q, que contêm um ou mais genes que codificam receptores relevantes para asma, inflamação, e desordens metabólicas, incluindo o gene receptor beta(2)-adrenérgico ADRB2 e o gene receptor glicocorticóide NR3C1.(46)

0 milieuinflamatório sistêmico da obesidade leva a complicações metabólicas e cardiovasculares, mas se esse ambiente altera o risco de asma ou fenótipo é ainda desconhecido. ${ }^{(46)}$ A leptina, uma citocina pró-inflamatória produzida pelo tecido adiposo, foi associada à asma em crianças..$^{(50)} \mathrm{A}$ recente literatura implica um papel pró-inflamatório para a hipercolesterolemia. Um estudo com 188 crianças e adolescentes mostrou que o nível sérico de colesterol foi maior no grupo de asmáticos do que no dos indivíduos saudáveis, e que os pacientes asmáticos eram significativamente mais obesos do que os não asmáticos. Neste estudo, a hipercolesterolemia e a obesidade aumentaram a probabilidade de asma independentemente. ${ }^{(51)}$

É importante entender o mecanismo da relação entre obesidade e asma, uma vez que a prevalência da obesidade é extremamente alta entre crianças que vivem em ambientes urbanos, onde a prevalência de asma é particularmente alta. ${ }^{(52)} \mathrm{Em}$ crianças em idade escolar e adolescentes, foi observada a associação entre hiperresponsividade da via aérea, atopia e IMC. ${ }^{(53)}$ No sul do Brasil, um estudo transversal com adolescentes mostrou associação positiva entre obesidade, prevalência, e severidade da asma, um achado predominante em meninas. ${ }^{(54)}$ A obesidade é um forte preditor de que a asma na infância seja recorrente na adolescência. ${ }^{(55)}$ Além disso, mais de 75\% dos pacientes que vão a um serviço de emergência para asma apresentam sobrepeso ou obesidade. ${ }^{(56)}$

0 estudo da relação obesidade-asma em grandes coortes, nas quais auto-relatos são freqüentemente usados para definir o diagnóstico de asma, é complicado pelas alterações na fisiologia pulmonar causadas pela obesidade, as quais podem levar à dispnéia ou a outros sintomas respiratórios, mas não preenchem os critérios fisiológicos aceitos para asma. ${ }^{(46)}$ As principais complicações respiratórias da obesidade incluem a demanda aumentada de oxigênio, o elevado trabalho respiratório, a ineficiência muscular respiratória, e complacência respiratória diminuída. A capacidade residual funcional e o volume de reserva expiratório diminuídos estão associados ao fechamento dos alvéolos 
periféricos, anormalidades na relação ventilaçãoperfusão e hipoxemia, especialmente na posição supina. ${ }^{(57)}$ Testes convencionais de função respiratória são pouco afetados pela obesidade, exceto em casos extremos. ${ }^{(57,58)}$ As principais complicações circulatórias são o maior volume sangüíneo total e pulmonar, alto débito cardíaco, e elevada pressão final diastólica do ventrículo esquerdo. Pacientes com obesidade comumente desenvolvem síndromes de hipoventilação e apnéia do sono, com atenuada responsividade das vias aéreas pela hipóxia, e hipercapnia. 0 resultado final é a hipoxemia, hipertensão pulmonar, e progressiva incapacidade. Pacientes obesos apresentam, com maior freqüência, sintomas de dispnéia, e menor capacidade de exercício, importantes para a qualidade de vida. Menos musculatura, mais dor articular, e fricção da pele, são importantes determinantes de menor capacidade de exercício, além dos efeitos cardiopulmonares da obesidade. Redução de peso e atividade física são meios efetivos para reverter essas alterações. ${ }^{(57)} 0$ diagnóstico e 0 manejo adequado do broncoespasmo induzido por exercício podem melhorar o desempenho de atividades físicas, ajudar na perda de peso, e quebrar o ciclo vicioso. ${ }^{(58)}$

\section{Considerações finais}

Nos últimos anos, o aspecto nutricional representa um importante fator condicionante para muitas doenças cardiovasculares, gastrointestinais, e pulmonares crônicas. Muitos trabalhos publicados documentam anormalidades inflamatórias específicas, nas vias aéreas de indivíduos com asma leve a moderada, sendo o estado inflamatório freqüentemente associado a uma maior geração de radicais livres de oxigênio. Essa evidência tem estimulado muitos pesquisadores a supor que o estresse oxidativo pode ser um importante fator patogênico, determinante na progressão de doenças crônicas, e que a diminuição dos insultos oxidantes ao pulmão pode ser modificada com terapia antioxidante suplementar. Suplementações dietéticas poderiam reduzir o estresse oxidante, minimizar o aparecimento de sintomas asmáticos, e constituir uma nova abordagem para o manejo da asma, em adição às estratégias farmacológicas correntemente em uso.

Em relação ao aleitamento materno, as evidências mais consistentes sugerem que não se deve diminuir o entusiasmo em recomendá-lo, na grande maioria das vezes. Não está claro como os mecanismos de imunomodulação expressam-se no binômio mãe-filho, e se é possível predizer como o leite materno afetará o desenvolvimento de alergias, em uma determinada criança. 0 entendimento de fatores genéticos pode permitir melhor previsibilidade desses desfechos, no futuro. Os novos estudos determinarão, também, o real efeito da complexa interação dos fatores imunomodulatórios entre mãe e filho, quanto ao leite materno, no desenvolvimento da doença alérgica.

Asma e obesidade têm significante impacto na saúde pública, e a incidência da asma tem sido associada à obesidade. 0 fato de asma e obesidade apresentarem, em alguns estudos, uma relação de associação significativa, não indica, necessariamente, uma relação de causa-efeito. A compreensão sobre os mecanismos dessa relação pode proporcionar o fundamento para a elaboração de novas estratégias terapêuticas direcionadas à população de risco, ao mesmo tempo em que se buscam alternativas para melhorar o controle e a qualidade de vida dos pacientes. Para isso, devem ser incorporadas intervenções sobre o estilo de vida, em que o papel da intervenção dietética deve ser avaliado quanto à redução da incidência de asma e atopia.

\section{Referências}

1. Heinrich J, Hoelscher B, Frye C, Meyer 1, Wjst M, Wichmann HE. Trends in prevalence of atopic diseases and allergic sensitization in children in Eastern Germany. Eur Respir J. 2002;19(6):1040-6.

2. Stick SM, Burton PR, Gurrin L, Sly PD, LeSouef PN. Effects of maternal smoking during pregnancy and a family history of asthma on respiratory function in newborn infants. Lancet. 1996;348(9034):1060-4.

3. Salam MT, Li YF, Langholz B, Gilliland FD. Maternal fish consumption during pregnancy and risk of early childhood asthma. J Asthma. 2005;42(6):513-8.

4. Kramer MS. Maternal antigen avoidance during pregnancy for preventing atopic disease in infants of women at high risk. Cochrane Database Syst Rev. 2000;(2):CD000133.

5. Oddy WH, Holt PG, Sly PD, Read AW, Landau Ll, Stanley FJ et al. Association between breast feeding and asthma in 6 year old children: findings of prospective birth cohort study. BMJ. 1999;319(7213):815-9.

6. Chulada PC, Arbes SJ, Dunson D, Zeldin DC. Breast-feeding and the prevalence of asthma and wheeze in children: analyses from the Third National Health and Nutrition Examination Survey, 1988-1994. J Allergy Clin Immunol. 2003;111(2):328-36

7. Kull 1, Wickman M, Lilja G, Nordvall SL, Pershagen G. Breast feeding and allergic diseases in infants-a prospective birth cohort study. Arch Dis Child. 2002;87(6):478-81. 
8. Kull 1, Almqvist C, Lilja G, Pershagen G, Wickman M. Breastfeeding reduces the risk of asthma during the first 4 years of life. J Allergy Clin Immunol. 2004;114(4):755-60.

9. Bergmann RL, Diepgen TL, Kuss 0, Bergmann KE, Kujat J, Dudenhausen JW et al. Breastfeeding duration is a risk factor for atopic eczema. Clin Exp Allergy. 2002;32(2):205-9.

10. Sears MR, Greene JM, Willan AR, Taylor DR, Flannery EM, Cowan J0, et al. Long-term relation between breastfeeding and development of atopy and asthma in children and young adults: a longitudinal study. Lancet. 2002;360(9337):901-7.

11. Hide DW, Guyer BM. Clinical manifestations of allergy related to breast and cows' milk feeding. Pediatrics. 1985;76(6):973-5.

12. Burgess SW, Dakin CJ, O'Callaghan MJ. Breastfeeding does not increase the risk of asthma at 14 years. Pediatrics. 2006;117(4):787-92.

13. Friedman NJ, Zeiger RS. The role of breast-feeding in the development of allergies and asthma. J Allergy Clin Immunol. 2005;115(6): 1238-48

14. Casas R, Böttcher MF, Duchén K, Björkstén B. Detection of $\lg A$ antibodies to cat, beta-lactoglobulin, and ovalbumin allergens in human milk. J Allergy Clin Immunol. 2000;105(6 Pt 1):1236-40.

15. Savilahti E, Siltanen M, Kajosaari M, Vaarala O, Saarinen KM. $\lg$ A antibodies, TGF-beta 1 and -beta2, and soluble CD14 in the colostrum and development of atopy by age 4 . Pediatr Res. 2005;58(6):1300-5

16. August A, Mueller C, Weaver V, Polanco TA, Walsh ER, Cantorna MT. Nutrients, nuclear receptors, inflammation, immunity lipids, PPAR, and allergic asthma. J Nutr. 2006;136(3):695-9.

17. Kalliomäki M, Ouwehand A, Arvilommi H, Kero P, lsolauri E. Transforming growth factor-beta in breast milk: a potential regulator of atopic disease at an early age. J Allergy Clin Immunol. 1999;104(6):1251-7.

18. Osterlund P, Smedberg T, Hakulinen A, Heikkilä H, Järvinen KM. Eosinophil cationic protein in human milk is associated with development of cow's milk allergy and atopic eczema in breast-fed infants. Pediatr Res. 2004;55(2):296-301.

19. Cant A, Marsden RA, Kilshaw PJ. Egg and cows' milk hypersensitivity in exclusively breast fed infants with eczema, and detection of egg protein in breast milk. Br Med J (Clin Res Ed). 1985;291(6500):932-5.

20. Troncone R, Scarcella A, Donatiello A, Cannataro P, Tarabuso A, Auricchio S. Passage of gliadin into human breast milk. Acta Paediatr Scand. 1987;76(3):453-6.

21. Saarinen KM, Juntunen-Backman K, Järvenpää AL, Klemetti $P$, Kuitunen $P$, Lope $L$, et al. Breast-feeding and the development of cows' milk protein allergy. Adv Exp Med Biol. 2000;(478):121-30.

22. Akobeng AK, Heller RF. Assessing the population impact of low rates of breast- feeding on asthma, coeliac disease and obesity: the use of a new statistical method. Arch Dis Child. 2006;92(6):483-5.

23. Lowe AJ, Carlin JB, Bennett CM, Abramson MJ, Hosking CS, Hill DJ, Dharmage SC. Atopic disease and breastfeeding--cause or consequence? J Allergy Clin Immunol. 2006;117(3):682-7.

24. Kelly FJ. Vitamins and respiratory disease: antioxidant micronutrients in pulmonary health and disease. Proc Nutr Soc. 2005;64(4):510-26.
25. Devereux G, Seaton A. Diet as a risk factor for atopy and asthma. J Allergy Clin Immunol. 2005;115(6):1109-17.

26. Mickleborough TD, Lindley MR, lonescu AA, Fly AD. Protective effect of fish oil supplementation on exercise-induced bronchoconstriction in asthma. Chest. 2006;129(1):39-49.

27. Pearson PJ, Lewis SA, Britton J, Fogarty A. Vitamin E supplements in asthma: a parallel group randomised placebo controlled trial. Thorax. 2004;59(8):652-6.

28. Fogarty A, Lewis S, Weiss S, Britton J. Dietary vitamin E, $\operatorname{lgE}$ concentrations and atopy. Lancet. 2000;356(9241):1573-4.

29. Patel BD, Welch AA, Bingham SA, Luben RN, Day NE, Khaw KT et al. Dietary antioxidants and asthma in adults. Thorax. 2006;61(5):388-93.

30. Ochs-Balcom HM, Grant BJ, Muti P, Sempos CT, Freudenheim JL, Browne RW, et al. Antioxidants, oxidative stress, and pulmonary function in individuals diagnosed with asthma or COPD. Eur J Clin Nutr. 2006;60(8):991-9.

31. Geronikaki AA, Gavalas AM. Antioxidants and inflammatory disease: synthetic and natural antioxidants with antiinflammatory activity. Comb Chem High Throughput Screen. 2006;9(6):425-42

32. Fogarty A, Britton J. The role of diet in the aetiology of asthma. Clin Exp Allergy. 2000;30(5):615-27.

33. Smit HA. Chronic obstructive pulmonary disease, asthma and protective effects of food intake: from hypothesis to evidence. Respir Res. 2001;2(5):261-4.

34. Romieu I, Varraso R, Avenel V, Leynaert B, Kauffmann F, Clavel-Chapelon F. Fruit and vegetable intakes and asthma in the E3N study. Thorax. 2006;61(3):209-15.

35. Garcia V, Arts IC, Sterne JA, Thompson RL, Shaheen SO. Dietary intake of flavonoids and asthma in adults. Eur Respir J. 2005;26(3):449-52.

36. Njå F, Nystad W, Lødrup Carlsen KC, Hetlevik 0, Carlsen KH. Effects of early intake of fruit or vegetables in relation to later asthma and allergic sensitization in school-age children. Acta Paediatr. 2005;94(2):147-54

37. Troisi RJ, Willett WC, Weiss ST, Trichopoulos D, Rosner B, Speizer FE. A prospective study of diet and adult-onset asthma. Am J Respir Crit Care Med. 1995;151(5):1401-8.

38. Shidfar F, Baghai N, Keshavarz A, Ameri A, Shidfar S. Comparison of plasma and leukocyte vitamin C status between asthmatic and healthy subjects. East Mediterr Health J. 2005;11(1-2):87-95.

39. Arora P, Kumar V, Batra S. Vitamin A status in children with asthma. Pediatr Allergy Immunol. 2002;13(3):223-6.

40. Wickens K, Barry D, Friezema A, Rhodius R, Bone N, Purdie $\mathrm{G}$, Crane J. Fast foods - are they a risk factor for asthma? Allergy. 2005;60(12):1537-41.

41. Cheng J, Pan T, Ye GH, Liu Q. Calorie controlled diet for chronic asthma. Cochrane Database Syst Rev. 2005;(3): CD004674.

42. McKeever TM, Britton J. Diet and Asthma. Am J Respir Crit Care Med. 2004;170(7):725-9.

43. Jackson RJ. The impact of the built environment on health: an emerging field. Am J Public Health. 2003;93(9):1382-4.

44. Mello E, Luft V, Meyer F. Obesidade Infantil:como podemos ser eficazes? J Pediatr (Rio J). 2004;80(3):173-82

45. Shore SA. Obesity and asthma: cause for concern. Curr Opin Pharmacol. 2006;6(3):230-6.

46. Beuther DA, Weiss ST, Sutherland ER. Obesity and asthma. Am J Respir Crit Care Med. 2006;174(2):112-9.

47. von Mutius E, Schwartz J, Neas LM, Dockery D, Weiss ST. Relation of body mass index to asthma and atopy in 
children: the National Health and Nutrition Examination Study III. Thorax. 2001;56(11):835-8.

48. Hancox RJ, Milne BJ, Poulton R, Taylor DR, Greene JM, McLachlan CR, et al. Sex differences in the relation between body mass index and asthma and atopy in a birth cohort. Am J Respir Crit Care Med. 2004;171(5):440-5.

49. Chinn S, Downs SH, Anto JM, Gerbase MW, Leynaert B, de Marco R, et al. Incidence of asthma and net change in symptoms in relation to changes in obesity. Eur Respir J. 2006;28(4):763-71.

50. Sood A, Ford ES, Camargo CA. Association between leptin and asthma in adults. Thorax. 2006;61(4):300-5.

51. Al-Shawwa B, Al-Huniti N, Titus G, Abu-Hasan M. Hypercholesterolemia is a potential risk factor for asthma. J Asthma. 2006;43(3):231-3.

52. Luder E, Melnik TA, DiMaio M. Association of being overweight with greater asthma symptoms in inner city black and Hispanic children. J Pediatr. 1998;132(4):699-703.

53. Jang AS, Lee JH, Park SW, Shin MY, Kim DJ, Park CS. Severe airway hyperresponsiveness in school-aged boys with a high body mass index. Korean J Intern Med. 2006;21(1):10-4.
54. Cassol VE, Rizzato TM, Teche SP, Basso DF, Centenaro DF, Maldonado M, et al. Obesity and its relationship with asthma prevalence and severity in adolescents from southern Brazil. J Asthma. 2006;43(1):57-60.

55. Guerra S, Wright AL, Morgan WJ, Sherrill DL, Holberg $\mathrm{CJ}$, Martinez FD. Persistence of asthma symptoms during adolescence: role of obesity and age at the onset of puberty. Am J Respir Crit Care Med. 2004;170(1):78-85.

56. Thomson CC, Clark S, Camargo CA; MARC Investigators. Body mass index and asthma severity among adults presenting to the emergency department. Chest. 2003;124(3):795-802.

57. Parameswaran K, Todd DC, Soth M. Altered respiratory physiology in obesity. Can Respir J. 2006;13(4):203-10.

58. Ulger Z, Demir E, Tanaç R, Gökşen D, Gülen F, Darcan S et al. The effect of childhood obesity on respiratory function tests and airway hyperresponsiveness. Turk $\mathrm{J}$ Pediatr. 2006;48(1):43-50. 